\title{
Using NLP in openEHR archetypes retrieval to promote interoperability: a feasibility study in China
}

Bo Sun ${ }^{1 \dagger}$, Fei Zhang ${ }^{1 \dagger}$, Jing Li ${ }^{2}$, Yicheng Yang ${ }^{3}$, Xiaolin Diao ${ }^{1}$, Wei Zhao ${ }^{1 *}$ (ID and Ting Shu ${ }^{4^{*}}$

\begin{abstract}
Background: With the development and application of medical information system, semantic interoperability is essential for accurate and advanced health-related computing and electronic health record (EHR) information sharing. The openEHR approach can improve semantic interoperability. One key improvement of openEHR is that it allows for the use of existing archetypes. The crucial problem is how to improve the precision and resolve ambiguity in the archetype retrieval.

Method: Based on the query expansion technology and Word2Vec model in Nature Language Processing (NLP), we propose to find synonyms as substitutes for original search terms in archetype retrieval. Test sets in different medical professional level are used to verify the feasibility.

Result: Applying the approach to each original search term $(n=120)$ in test sets, a total of 69,348 substitutes were constructed. Precision at 5 (P@5) was improved by 0.767, on average. For the best result, the P@5 was up to 0.975.

Conclusions: We introduce a novel approach that using NLP technology and corpus to find synonyms as substitutes for original search terms. Compared to simply mapping the element contained in openEHR to an external dictionary, this approach could greatly improve precision and resolve ambiguity in retrieval tasks. This is helpful to promote the application of openEHR and advance EHR information sharing.
\end{abstract}

Keywords: OpenEHR, Nature language processing, Information retrieval, Interoperability

\section{Background}

With the development of big data processing technology, the effective use of medical data inevitably became a trend [1]. The improvement of the quality of medical services, the reduction of medical service costs, and even

*Correspondence: zw@fuwai.com; nctingting@126.com

${ }^{\dagger}$ Bo Sun and Fei Zhang have contributed equally to this work ${ }^{1}$ Department of Information Center, Fuwai Hospital, National Center for Cardiovascular Diseases, Chinese Academy of Medical Sciences and Peking Union Medical College, No. 167 North Lishi Road, Xicheng District, Beijing 100037, China

${ }^{4}$ National Institute of Hospital Administration, National Health Commission, Building 3, yard 6, Shouti South Road, Haidian, Beijing 100044, China

Full list of author information is available at the end of the article the progress and development of medicine have become increasingly dependent on the effective use of medical data [2-4]. More recently, the Electronic Health Record (EHR) has been defined as a viable source of data for regulatory decision-making [5]. However, bias can occur along the various steps of the data chain, and can lead to unusable data or invalid analysis results [6]. The lack of semantic interoperability is cited as a primary reason for inefficiencies within the healthcare system in the United States, contributing to billions of wasted dollars annually $[7,8]$. Thus, semantic interoperability [9] is essential for accurate and advanced health-related computing and EHR sharing [10,11]. Medical information models are used to improve semantic interoperability $[12,13]$. 
Currently, main-stream medical information models about semantic interoperability include: HL7-V3 [14], FHIR [15], ISO13606 [16-18], openEHR [19], and more. In this regard, openEHR is of particular interest because a large community of developers and many open-source tools are available [20]. OpenEHR has already been implemented in several countries (e.g. the United Kingdom, Australia) and is attractive to developing countries [21-23]. The Medical Software Branch and the Smart and Mobile Medical Branch of the China Association for Medical Devices Industry jointly established openEHR China in March 2016. In 2018, Min et al. built Chinese archetypes that were uploaded to the Healthcare Modelling Collaboration (HMC) [24]. HMC manages archetypes and facilitates the reuse of the same archetypes in China.

The openEHR approach can improve semantic interoperability $[25,26]$. One key improvement in openEHR compared to other systems is that, as the name 'open' implies, it allows for the use of both existing and newly created archetypes [27]. Archetype plays an important role in the openEHR approach, as it supports not only semantics but also scalability and interoperability [25]. The crucial problem is finding the relevant archetypes from open repositories [28]. It is difficult to achieve this goal. These concept names of archetypes are described by professional medical terms [25]; however, some users, including patients [29], may use layperson wording for terms when searching target archetypes [30]. Similar to searching in PubMed, the most common reason for retrieval error is a lack of synonymous terms [32].

In recent years, the importance of synonym-learning which may help alleviate the lack of synonyms is well recognised in the NLP research community, especially in the biomedical [30] and clinical domains [31]. The most important part of synonym-learning is semantic extraction. With the development of NLP technology such as multi-label text categorization [32], text generation [33] and so on, there are many researches about semantic extraction. Yang et al. [34] combined reinforcement learning, generative adversarial networks, and recurrent neural networks to build a termed category sentence generative adversarial network (CS-GAN), which can help to generate synonymous sentences so that enlarge the original dataset. Younas et al. [35] manipulated the textual semantic of functional requirements to identify the nonfunctional requirements in software development and used the similarity distance between the popular indicator keywords and requirement statements to identify the type of non-functional requirement. In their study, the semantic similarity is calculated based on co-occurrence of patterns in large human knowledge repositories of Wikipedia. Liu et al. [36] proposed a novel end-to-end multi-level semantic representation enhancement network (MLSREN) which can enhance the semantic representation of entities from word, phrase, and context level. With the semantic representation of words, we can calculate the similarity between them, and find synonymous terms which can be used to expand the user's search terms and realize query expansion.

Crimp et al. [37] used the semantic dictionary WordNet to expand the query, then refined the candidate expansions by discriminating relevancy and excluding spurious expansion terms which help reduce query drift and increase query performance. ItiChaturvedi et al. proposed a Variable-order Belief Network (VBN) framework, which is good at modeling word dependencies in text, can be used for semantic representation of words [38]. Similarly, Huang et al. [39] used the deep belief network (DBN) model to capture the meaningful terms for effective query expansion in the code searching task. The model both extracts relevant terms to expand a query and excludes irrelevant terms from the query and outperforms several query expansion algorithms for code search. Yusuf et al. [40] enhanced the query expansion method based on unigram model and word embedding using Glove, which can capture the semantic similarity. The results show that Glove's [41] model for word embedding can significantly improve query expansion methods using Arberry dataset. Another famous Word embedding model is Word2Vec, developed by Mikolov et al. [41] which represents words with a continuous vector obtained from a neural network model trained on a huge text corpus. Since the word vector contains the semantic information of the word, the similarity between the two words is accurately reflected by the distance of the word vector. At present, Word2Vec is widely used in the calculation of word similarity.

Aim of this study is resolve mistakes related to ambiguity and promote semantic interoperability. Therefore, we proposed and assessed an approach using NLP technology and corpus to expand search terms by finding synonyms as alternative terms. We also sought to verify the process by testing examples taken from Chinese archetypes and corpus.

\section{Methods \\ Approach description}

For an original search term, we use the query expansion technology to find its synonyms as a substitute to search the target archetype in openEHR (Fig. 1). By using this in archetype retrieval, we can choose dictionaries or corpus in different fields to expand the search terms entered by people who with different backgrounds. This ability is essential for improving openEHR's interactivity, retrieval precision and application in different regions 


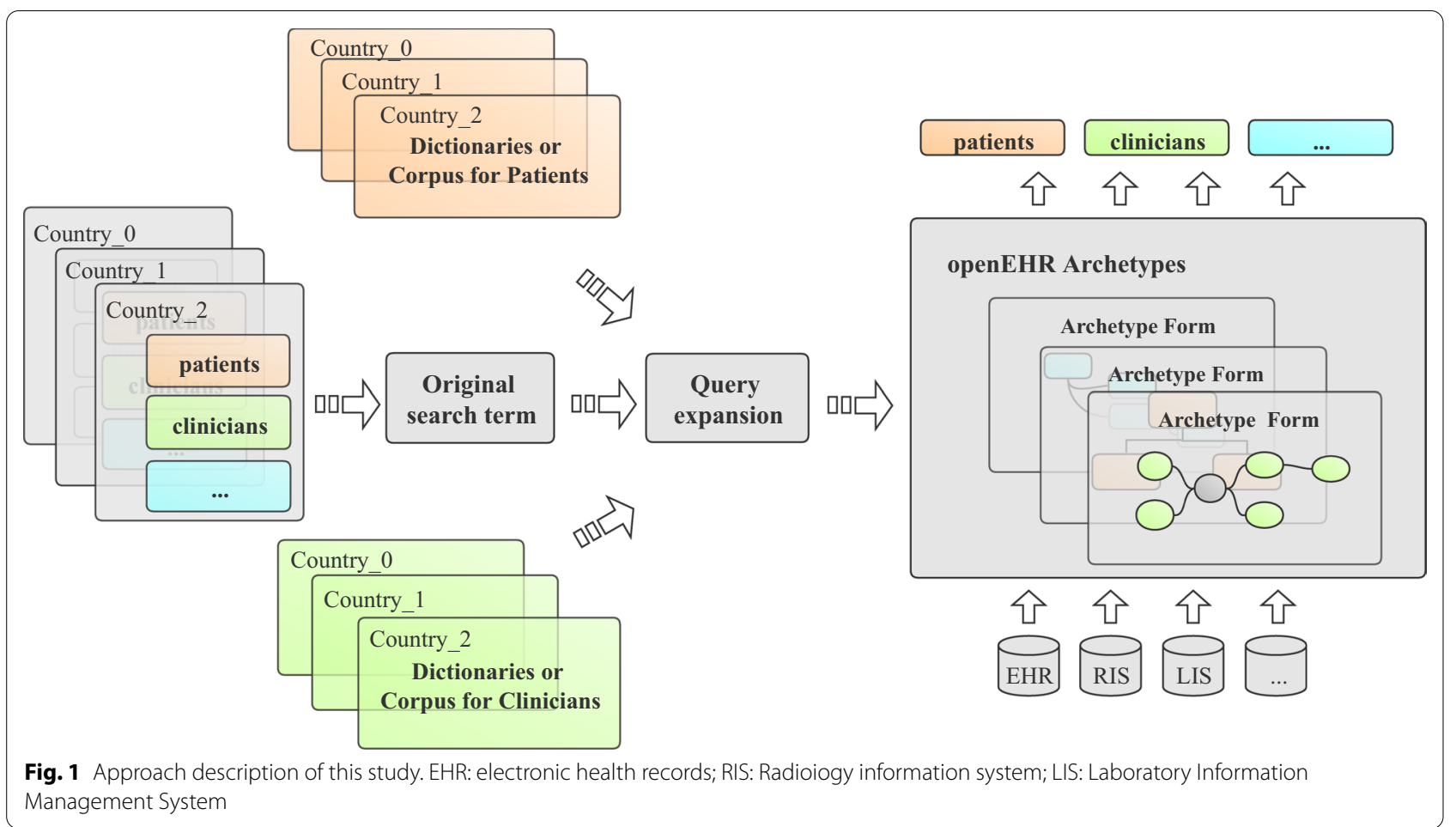

or countries. Such as expanding radiologist search terms by RadLex, expanding search terms of clinicians by Unified Medical Language System (UMLS) Metathesaurus, Systematized Nomenclature of Medicine Clinical Terms (SNOMED-CT) and expanding search terms used by patients (people who without medical knowledge) by Word-Net, Wikipedia, and so forth. Using the dictionaries and corpora of different countries also allows us to expand the search terms entered in different countries.

There are three key steps of query expansion: term segmentation, term expansion and term combination (Fig. 2). Term segmentation is dividing the original search term into a sub term. Term expansion is the use of NLP technology and materials to further provide synonyms for the sub terms. Term combination is to combine expansion terms to form combination terms. Finally, expansion terms and combination terms are used as substitutes of the original search term to search archetypes in openEHR.

To prove the feasibility of the approach, we selected the Chinese openEHR archetypes as data sources, built test sets and chose the Chinese Wikipedia data as the expanded corpus.

\section{Data sources}

The Chinese archetypes are stored by HMC. Having only 64 archetypes when it was created in 2018, but now it includes 410 archetypes. Among these, 16 concept names of archetypes are described only in English, so we used the other 394 archetypes as the data source.

\section{Test sets}

In order to simulate the real input search term to the greatest extent, we constructed test sets with different medical professional levels: low, medium and high. The construction follows these principles: first, search terms should be relevant to the Chinese EHR; second, search terms should reuse some clinical content, such as medical events prediction, clinical research and disease research. We defined the content contained in the test sets as original search terms, a total of 45 original search terms was constructed (Low Level Set:15, Medium Level Set:15, High Level Set:15). The directly searching of target archetypes in HMC with original search term was definded as baseline mode (Fig. 3).

\section{Test set with low medical professional level (Low Level Set)}

We divided all archetypes stored in the data source (published in HMC) into three parts: basic patient information, medication and clinical examination. For each part, we randomly selected five archetypes, a total of 15 target archetypes. We used 'HIT IR-Lab Tongyici Cilin extended version (TC-E)' [42] to find related synonyms of target archetypes' Chinese names as original search terms. TC-E is an authoritative semantic dictionary in the common languages field of Chinese [43]. For example, 'health 
A

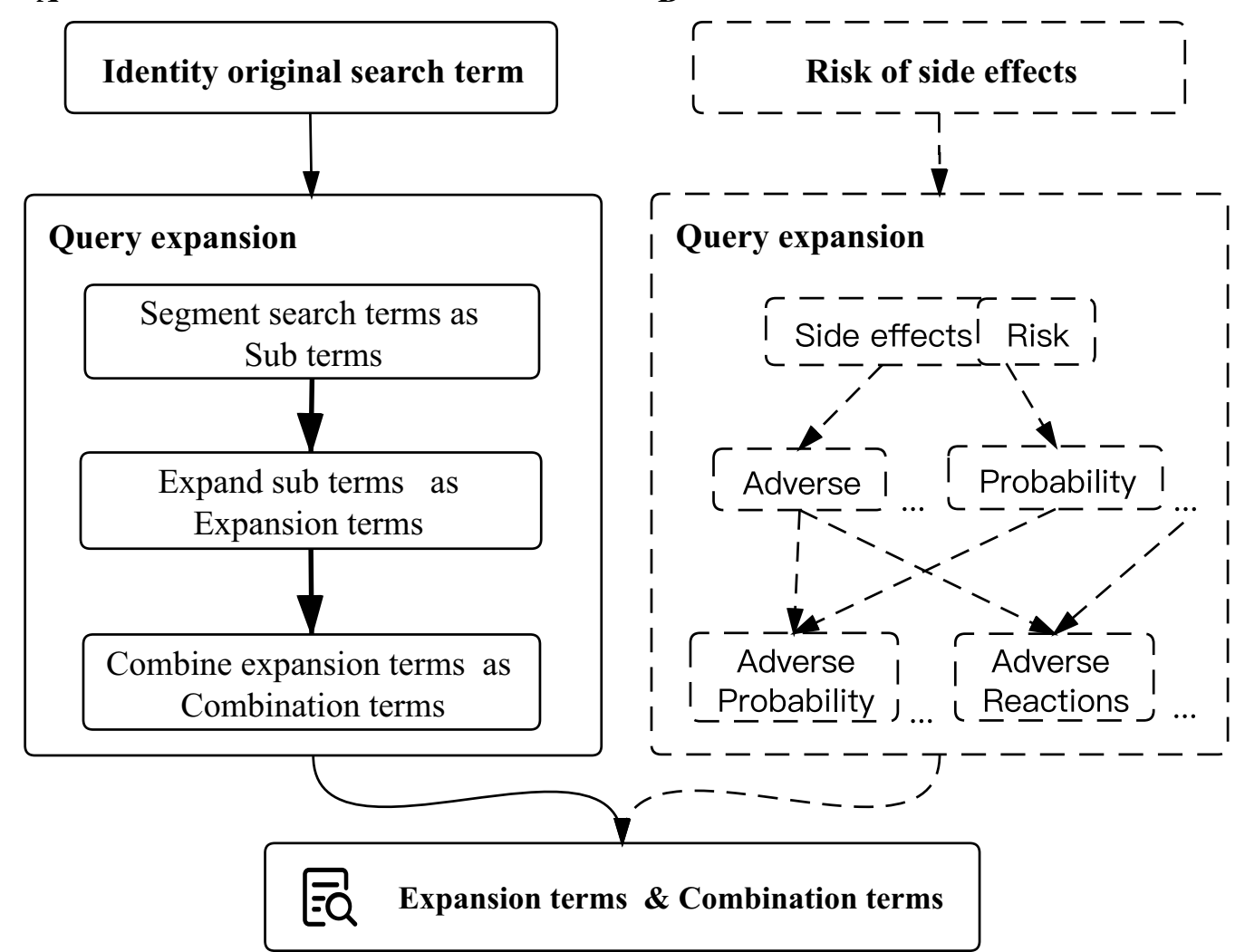

Fig. 2 Process of query expansion. a Three key steps of query expansion: term segmentation, term expansion and term combination; $\mathbf{b}$ Example of query expansion: risk of side effects

summary' is the Chinese name of archetype openEHREHR-COMPOSITION-health-summary.v1. Searching in TC-E, we found that 'health abstract' is a synonym of it. Hence, 'health abstract' was used as original search terms for archetype openEHR-EHR-COMPOSITION. healthsummary.v1. In the end, a total of 40 terms was generated (Table 1). Since TC-E is a collection of common Chinese languages, we assumed this test set's medical professional level is 'low'

\section{Test set with medium medical professional level (Medium Level Set)}

For better contrast, we chose the target archetypes in Low Level Set as the target archetypes. We searched these archetypes' Chinese names in online search engines (Bing, Google, etc.) to find related terms as original search terms. For example, we searched 'fetal heartrate', the content name of the archetype openEHREHROBSERVATION. fetal heart.v1 in Bing and got medical science articles contained 'fetal heartbeat' [10]. Hence, we use 'fetal heartbeat' as original search terms for the archetype
openEHREHROBSERVATION.fetal heart.v1. Repeating the above operations, a total of 40 terms were found (Table 1). Because the test set is composed of related terms in popular science articles and medical Q\&A websites data, we assumed its medical professional level was 'medium'.

\section{Test set with high medical professional level (High Level} Set).

By searching 'ehr' or 'emr' in PubMed, we screened the literature with relevant data published over a period of 20 years and found the EHR data provided by relevant studies. Combining with the EHR data mentioned in the literature and the 'Basic Data Set of Electronic Health Record' issued by the China Health Construction Commission, we chose some content names of the EHR as original search terms to construct the test set (Table 1). Because the test set is composed of element names in EHR, we assume its medical professional was 'high'. 


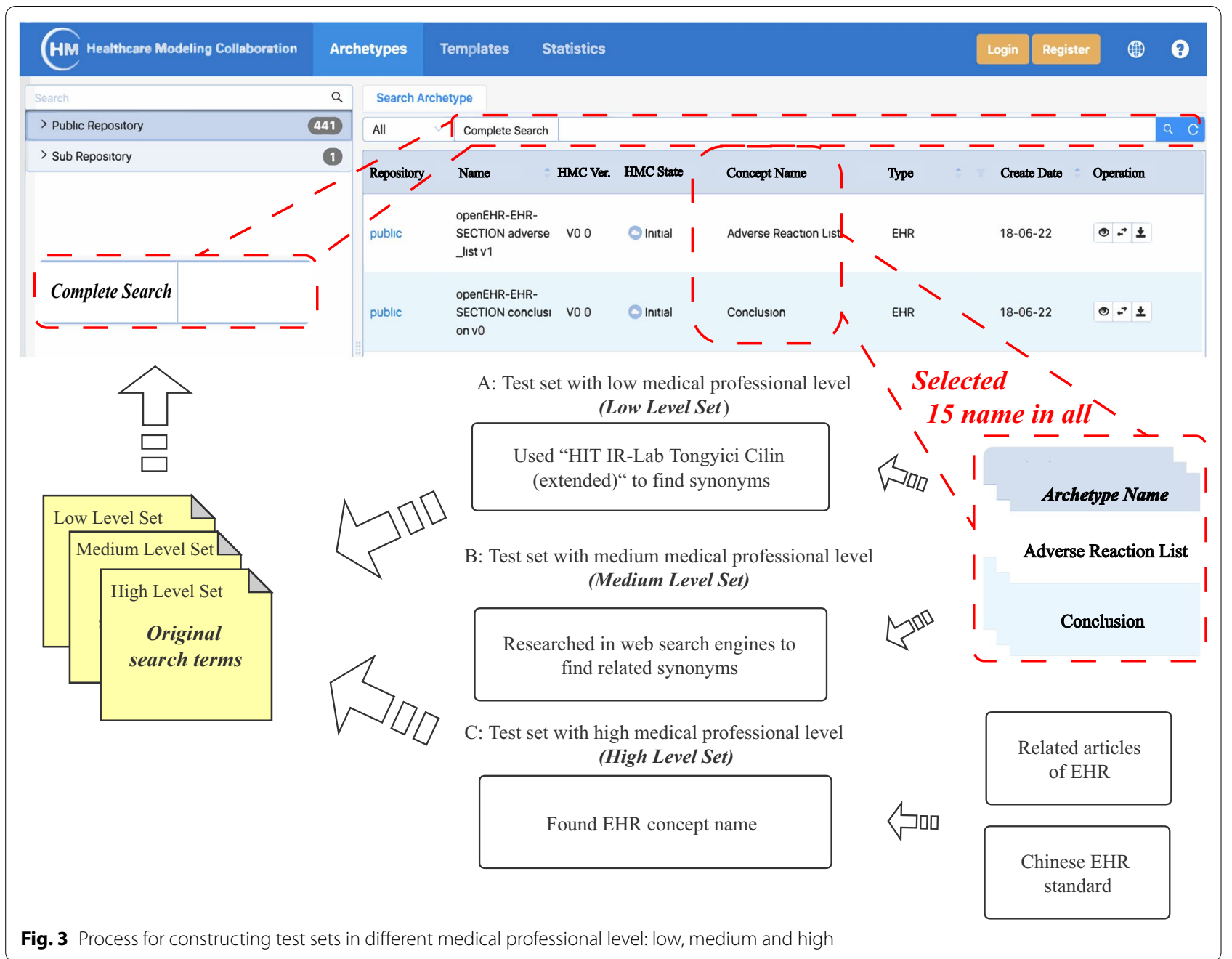

\section{NLP and query expansion}

Query expansion (popular in text retrieval community) is a technique used to improve search precision. The basic idea is using results from an initial query to reformulate the query and then get more precise results. Data sources used in query expansion methods rely on external lexicalsemantic resources, typically dictionaries or other similar knowledge representation resources.

Table 1 Description of test sets

\begin{tabular}{|c|c|c|c|c|}
\hline \multirow[t]{2}{*}{ Test sets } & \multicolumn{2}{|l|}{ Target archetype } & \multicolumn{2}{|l|}{ Original search terms } \\
\hline & Examples & Number, $\mathrm{n}$ & Examples & Number, $n$ \\
\hline Low level set & $\begin{array}{l}\text { Skeletal age, medicine } \\
\text { management, etc }\end{array}$ & 15 & Bone age, drug management, etc & 40 \\
\hline Medium level set & $\begin{array}{l}\text { Fetal heartrate, care pro- } \\
\text { gram, etc }\end{array}$ & 15 & Fetal heartbeat, care plan, etc & 40 \\
\hline High level set & NA & NA & $\begin{array}{l}\text { Blood transfusion label, admission diagnosis, } \\
\text { etc }\end{array}$ & 40 \\
\hline
\end{tabular}

\section{Term segmentation}

Word segmentation is an important preprocessing step in several NLP systems [44] such as machine translation [45], information retrieval [46], etc. Existing word segmentation algorithms can be roughly divided into four categories [47]: manual rules-based, dictionariesbased, traditional machine learning-based and deep learning-based. Considering the complexity of the system and segmentation precision, in this study, we chose 
the word-segmentation algorithm based on traditional machine learning to accomplish the segmentation task.

\section{Term expansion}

Synonym expansion is the next step for resolving ambiguity and improving the precision of the archetype retrieval after segmenting the original terms. In the medical field, there is currently no high-quality lexicon of Chinese synonyms. Word embedding technology that has emerged in recent years is expected to solve this problem, which can express words as continuous vectors - the distance between two words in space can indicate the semantic connection or similarity of them. Word2Vec [48], uses context information for training, maps words into a high-dimensional space and uses the distance in the high-dimensional space as the basis for calculating the semantic similarity between two words. Therefore, at the algorithm level, retrieval is based on more 'semantic distance' than 'rule matching. Based on this idea, we aimed to implement synonym-expansion based on the Word2Vec trained by the Wikidata-corpus (Fig. 4).

\section{Assessment}

HMC provide two search methods: 'Normal search' and 'Completes search'. We choosed 'Completes search' as search method since it could search more content.

For Low and Medium Level Set, since the original search terms are derived from the 15 target archetypes that have been selected in HMC, we could compare the search result return by HMC with target archetypes to determine which is true. Meanwhile, for each original search term, there were many related expansion and combination terms (Tabel 4), and multiple search results. In this condition, we use Word2vec to calculate the semantic similarity of each results' archetype name and original search term. Sorting these results in descending order, according to the relevance (Fig. 5, Assessment method A).

For High Level Set, due to these original search terms are derived from EMR data, we did not know which

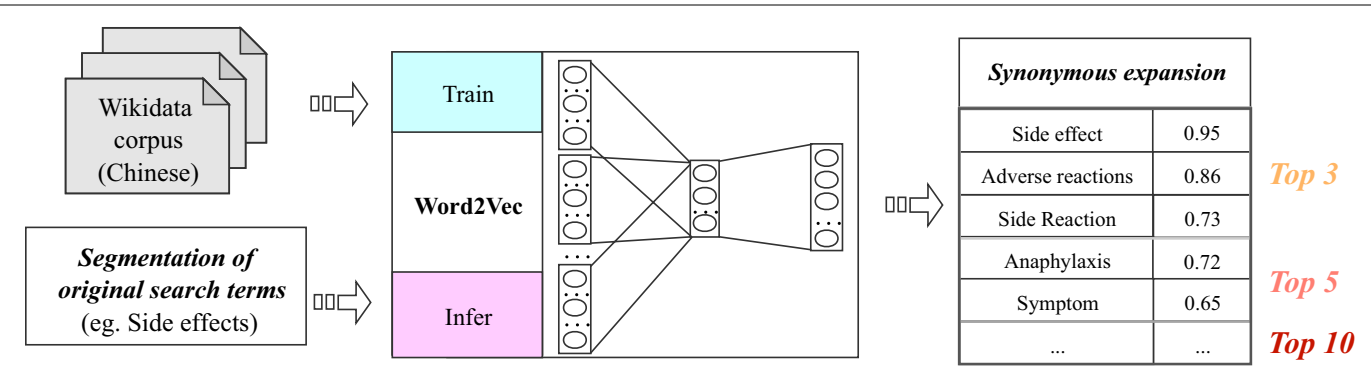

Fig. 4 The illustration of synonym-expansion based on the Word2Vec. At train stage, Wikidata in Chinese is used to learn the representation vector of words, and then use the cosine distance to calculate the similarity between user's term with all terms in vocabulary, after ranking, return top 10 terms as the expansion of user's term

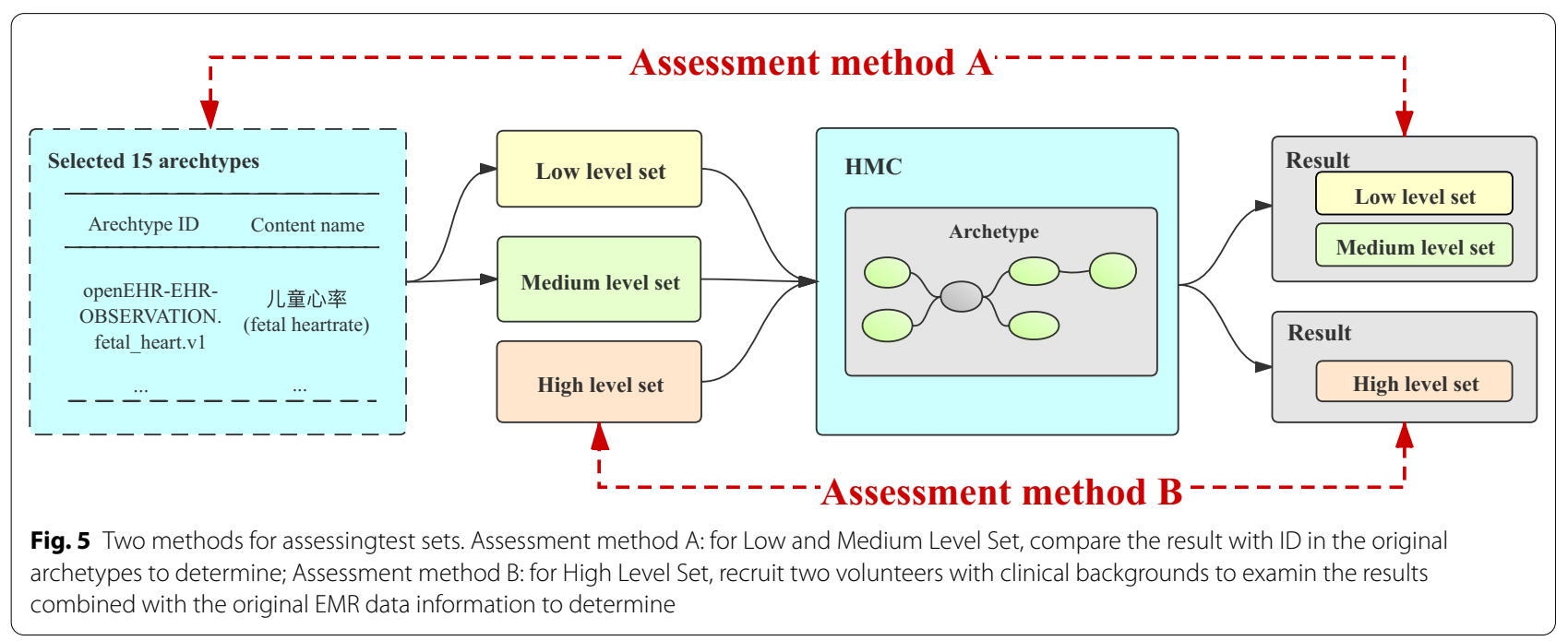


search result is true. In this condition, two volunteers with clinical backgrounds were recruited. They examined the results combined with the original EMR data. A total of 5 part needed to be manually assessment, especially, results of original search terms, results of expansion terms (Top3 and 5) and results of combination terms (Top3 and 5). Two volunteers assessed these results, back to back. For the inconsistent assessments, the two sides discussed and got the final result (Fig. 5, Assessment method B).

Precision at $\mathrm{K}(\mathrm{P} @ \mathrm{~K})$, average precison (AP) and mean average precision (MAP) are used as evaluation metrics. The precision at $\mathrm{K}(\mathrm{P} @ \mathrm{~K})$ is calculated as follows:

$$
P @ K=\frac{\sum_{i=0}^{N} \delta(i, K)}{N}
$$

$\delta(i, K)$ is an indicator function which equals 1 , if there is an acceptable result among the top $K$ returned by HMC or equals $0 . N$ is the total number of test set (low level test: 40, medium level test: 40, high level test: 40). In this study, $\mathrm{K}$ is 3 and 5 respectively (P@3, P@5).
The average precision (AP) is a measure that combines recall and precision for ranked retrieval archetypes. For one test set, AP is the mean of the P@r after top $r$ relevant archetypes are retrievel for each search term (origianl search terms or expansion terms or combination terms), $r \in\{3,5\}$.

$$
A P=\frac{1}{R} \sum_{r} P @ r
$$

The mean average precision (MAP) is the arithmetic mean of the AP values for a retrieval system over a set of $n$ test sets. $n \in\{$ low level set, medium level set, high level set $\}$. It can be expressed as follows:

$$
M A P=\frac{1}{N} \sum_{n} A P_{n}
$$

\section{Results}

\section{Overview of expansion results}

For each original search terms in the test sets, in the process of synonym expansion, the first 3, 5, and 10 terms

\begin{tabular}{|c|c|c|c|c|}
\hline \multirow[t]{2}{*}{ Original search term } & \multirow[t]{2}{*}{ Segmentation terms } & \multicolumn{3}{|l|}{ Expansion terms } \\
\hline & & Top 3 (sum: 6) & Top 5 (sum: 10) & Top 10 (sum: 20) \\
\hline \multirow[t]{10}{*}{ Risk of side effects } & Side effects & Side effect & Side effect & Side effect \\
\hline & & Adverse reactions & Adverse reactions & Adverse reactions \\
\hline & & $\ldots$ & Untoward reactions & Untoward reactions \\
\hline & & & $\ldots$ & Anaphylaxis \\
\hline & & & & $\ldots$ \\
\hline & Risk & Risk & Risk & Risk \\
\hline & & Possibility & Possibility & Possibility \\
\hline & & $\ldots$ & Danger & Danger \\
\hline & & & $\ldots$ & Probability \\
\hline & & & & $\ldots$ \\
\hline
\end{tabular}
of similar results were used as expansion terms and were

Table 2 Process for constructing expansion terms (for example: risk of side effects)

Table 3 Process for constructing combination terms (for example: risk of side effects)

\begin{tabular}{llll}
\hline Original search term & \multicolumn{2}{l}{ Combination terms } & Top 10 (sum: 100) \\
\cline { 2 - 4 } & Top 3 (sum: 9) & Top 5 (sum: 25) & Side effect risk \\
\hline Risk of side effects & Side effect risk & Side effect risk & Side effect possibility \\
& Side effect possibility & Side effect possibility & Adverse reactions risk \\
$\ldots$ & Adverse reactions risk & Adverse reactions possibility & Adverse reactions possibility \\
& $\ldots$ & Untoward reactions risk \\
& & Untoward reactions Possibility \\
\end{tabular}


combined into combination terms, which are recorded as Top 3, Top 5, and Top 10. Take the original search terms 'Risk of side effects' as an example: Table 2 shows the construction process of its expansion terms; Table 3 shows the construction process of its combination terms. For all 120 original search terms, a total of 4729 expansion terms and 64,619 combination terms were constructed (Table 4).

\section{Evaluation of the performance}

Manual assessment results of High Level Set are shown in Table 5. From the table, we can see that the two volunteers did agree on the search results of original search terms but difference on that of expansion and combination terms. After discussion again, finally result is obtained as the result of High Level Set.

Expansion terms achieved the best mean MAP (0.819) with an MAP of $0.967,0.883,0.608$ for each test set respectively. Combination terms achieved the mean MAP of 0.317, with an MAP of 0.567, 0.217, 0.167 for each test set respectively (Fig. 6a). For the same expansion term, the search results with different thresholds (Top3, 5, 10) are also different. According to Table 6, when the threshold is Top 3, AP $=0.963$ (Low Level Set), 0.888 (Medium Level Set) and 0.637 (High Level Set). In contrast, when the threshold is Top 10, AP $=0.961$ (Low Level Set), 0.875 (Medium Level Set) and 0.608 (High Level Set) Table 6.
In this article, $\mathrm{K}$ in the Formula (1) is 3 and 5 respectively, and get P@3, P@5. From Table 6, we can know that P@5 greater than P@3 on each test set. Taking Low Level Set for example: mean $\mathrm{P} @ 5=0.983$ (Expansion terms) and 0.583 (Combination terms), mean $\mathrm{P} @ 3=0.949$ (Expansion terms) and 0.550 (Combination terms).

By selecting Expansion terms and Top 3 as the best parameters, this method is compared with the baseline way (that is, directly use original search terms of test sets to search archetype, without any processing), which could show the superiority of this method. The results are shown in Fig. 6b. Firstly, there is no big gap among the search results of baseline method in three test set, as they are all lower than 0.2 (P@5). After using the method (Expansion Terms and Top 3), P@5 of each test set has been greatly improved. In Low Level Set, P@5 is the highest (0.975) and in contrast, High Level Set get the worst result: P@5=0.750. Compare to the baseline, the result of each data set is significantly improved. In Low Level Set, after using the method presented, P@5 is increased by 0.925 . The same is for other two set, which are increased by 0.775 and 0.600 , respectively.

\section{Disscusion}

In order to verify this method, we used three different methods to construct test sets with different medical professional level: Low, Medium and High. P@3, P@5,

Table 4 Results for constructing expansion terms \& combination terms

\begin{tabular}{|c|c|c|c|c|c|c|c|}
\hline \multirow[t]{2}{*}{ Test queries } & \multirow{2}{*}{$\begin{array}{l}\text { Original search } \\
\text { term, } n\end{array}$} & \multicolumn{3}{|c|}{ Expansion term, $\mathrm{n}$} & \multicolumn{3}{|c|}{ Combination term, $\mathrm{n}$} \\
\hline & & Top 3 & Top 5 & Top 10 & Top 3 & Top 5 & Top 10 \\
\hline Low level set & 40 & 216 & 360 & 719 & 312 & 840 & 3279 \\
\hline Medium level set & 40 & 267 & 455 & 891 & 546 & 2085 & 13,712 \\
\hline High level set & 40 & 303 & 505 & 1013 & 1050 & 6300 & 36,486 \\
\hline
\end{tabular}

Table 5 Manual assessment results of 'High Level Set'

\begin{tabular}{|c|c|c|c|c|c|c|c|c|c|c|}
\hline \multirow[t]{2}{*}{ Methods } & & \multicolumn{3}{|c|}{ Volunteer A } & \multicolumn{3}{|c|}{ Volunteer B } & \multicolumn{3}{|c|}{ Finally result } \\
\hline & & AP & P@3 & P@5 & AP & P@3 & P@5 & AP & P@3 & P@5 \\
\hline \multirow[t]{3}{*}{ Original search terms } & Top 3 & 0.150 & & & 0.150 & & & 0.150 & & \\
\hline & Top 5 & & & & & & & & & \\
\hline & Top 10 & & & & & & & & & \\
\hline \multirow[t]{3}{*}{ Expansion terms } & Top 3 & 0.637 & 0.525 & 0.750 & 0.662 & 0.575 & 0.750 & 0.637 & 0.525 & 0.750 \\
\hline & Top 5 & 0.575 & 0.475 & 0.675 & 0.612 & 0.550 & 0.675 & 0.612 & 0.550 & 0.675 \\
\hline & Top 10 & 0.575 & 0.500 & 0.650 & 0.575 & 0.500 & 0.650 & 0.575 & 0.500 & 0.650 \\
\hline \multirow[t]{3}{*}{ Combination terms } & Top 3 & 0.150 & 0.150 & 0.150 & 0.175 & 0.175 & 0.175 & 0.150 & 0.150 & 0.150 \\
\hline & Top 5 & 0.150 & 0.150 & 0.150 & 0.175 & 0.175 & 0.175 & 0.175 & 0.175 & 0.175 \\
\hline & Top 10 & 0.150 & 0.150 & 0.150 & 0.200 & 0.200 & 0.200 & 0.175 & 0.175 & 0.175 \\
\hline
\end{tabular}

Finally result values are emphasized in bold 
a

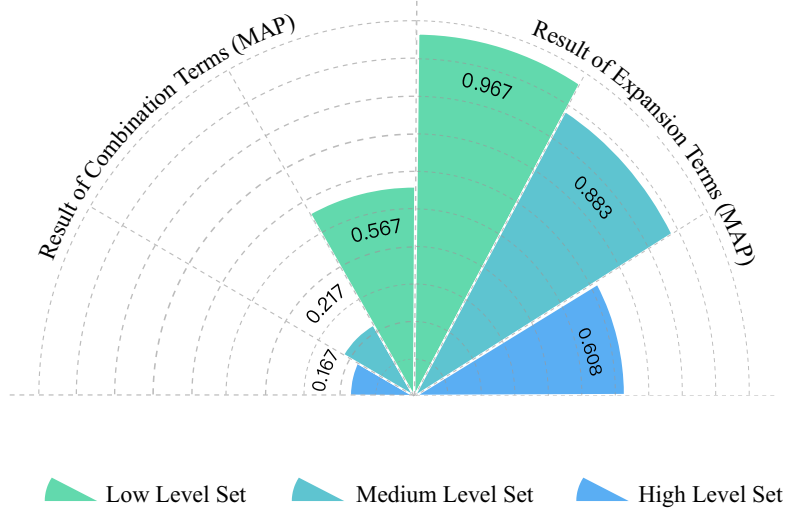

b

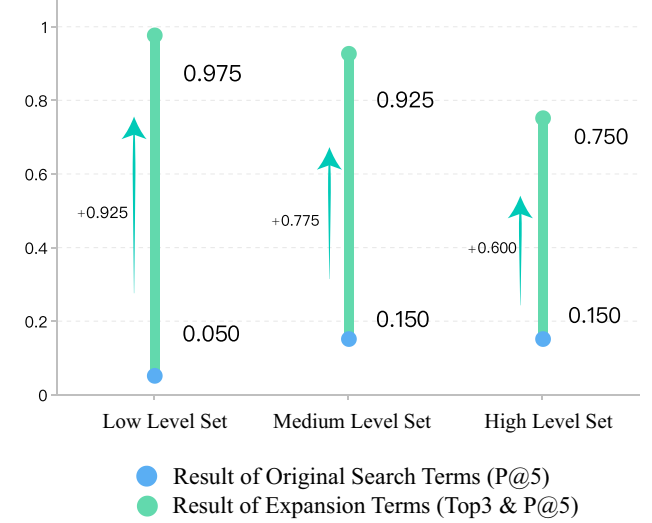

Fig. 6 Comparison result with baseline model. a Comparison result between expansion term and combination terms. $\mathbf{b}$ Comparison result between our method (Expansion terms \& Top 3) and baseline model

Table 6 Retrieval performance comparison between expansion terms and combination terms with different similarity thresholds

\begin{tabular}{|c|c|c|c|c|c|}
\hline \multicolumn{3}{|l|}{ Methods } & \multirow{2}{*}{$\begin{array}{l}\text { AP } \\
0.050\end{array}$} & \multirow{2}{*}{$\begin{array}{l}\text { P@3 } \\
0.050\end{array}$} & \multirow{2}{*}{$\begin{array}{l}\text { P@5 } \\
0.050\end{array}$} \\
\hline Low level set & Original search terms & & & & \\
\hline & Expansion terms & Top3 & 0.963 & 0.950 & 0.975 \\
\hline & & Top5 & 0.975 & 0.950 & 1.000 \\
\hline & & Top10 & 0.963 & 0.950 & 0.975 \\
\hline & & Mean & 0.967 & 0.950 & 0.983 \\
\hline & Combination terms & Top3 & 0.512 & 0.500 & 0.525 \\
\hline & & Top5 & 0.575 & 0.550 & 0.600 \\
\hline & & Top10 & 0.6125 & 0.600 & 0.625 \\
\hline & & Mean & 0.567 & 0.550 & 0.583 \\
\hline \multirow[t]{9}{*}{ Medium level set } & Original search terms & & 0.137 & 0.125 & 0.15 \\
\hline & Expansion terms & Top3 & 0.888 & 0.850 & 0.925 \\
\hline & & Top5 & 0.888 & 0.850 & 0.925 \\
\hline & & Top10 & 0.875 & 0.850 & 0.900 \\
\hline & & Mean & 0.883 & 0.850 & 0.917 \\
\hline & Combination terms & Top3 & 0.200 & 0.200 & 0.200 \\
\hline & & Top5 & 0.200 & 0.200 & 0.200 \\
\hline & & Top10 & 0.250 & 0.250 & 0.250 \\
\hline & & Mean & 0.217 & 0.217 & 0.217 \\
\hline \multirow[t]{9}{*}{ High level set } & Original search terms & & 0.150 & 0.150 & 0.150 \\
\hline & Expansion terms & Top3 & 0.637 & 0.525 & 0.750 \\
\hline & & Top5 & 0.612 & 0.550 & 0.675 \\
\hline & & Top10 & 0.575 & 0.500 & 0.650 \\
\hline & & Mean & 0.608 & 0.525 & 0.692 \\
\hline & Combination terms & Top3 & 0.150 & 0.150 & 0.150 \\
\hline & & Top5 & 0.175 & 0.175 & 0.175 \\
\hline & & Top10 & 0.175 & 0.175 & 0.175 \\
\hline & & Mean & 0.167 & 0.167 & 0.167 \\
\hline
\end{tabular}

Different similarity thresholds: in the process of synonym expansion, the first 3,5 , and 10 terms of similar results are used as expansion terms and then composed as combination terms

AP: average precision, $\mathrm{P} @ 3$ : precision at 3, P@5: precision at 5
AP and MAP are used as evaluation metrics. In this study, for a search term, if the target archetype appears in the first three results returned by HMC, then P@3 is 1. If the target archetype appears in the first five, then P@5 is 1 . Generally speaking, for a retrieval method, P@5 will be greater than P@3[49], the same is true in our study. Baseline model, which directly uses the original search terms for retrieval, got the lowest precision on the three test sets. On the P@5 level, only 2, 6 and 6 original search terms can search the target archetype, respectively. The low precision is caused by the mismatch between the search term and the name of the openEHR archetype. With the improvement of the medical professional level of search term, the search precision has also been improved. AP is 0.050 (Low level set), 0.137 (Medium level set) and 0.150 (High level set) respectively.

The purpose of this study is to promote the interoperability in the using of openEHR. Choosing Expansion terms and Top 3 as the best parameters to compare with baseline, P@3 and P@5 are increased by $90.0 \%$ and 92.5\% (Low Level Set), 72.5\% and 77.5\% (Medium Level Set), $37.5 \%$ and $60.0 \%$ (High Level Set). The improvement of different test set is also different. P@5 of Low Level Set is up to 0.975 and of High Level Set is up to 0.750 . The difference may be due to Chinese Wikipedia data. Wikipedia is not a professional medical corpus but does contain various kinds of medical vocabulary terms. It is easy to establish a connection between professional and lay medical vocabularies for people without medical knowledge.

Regarding the test sets, Mean P@5 is improved by 0.767. It has been proven that this method has superiority and generality. This is very meaningful, for that even in the same country, the distribution of medical resources and the level of doctors are often unbalanced. Take China 
as an example, the level of knowledge of medical staff in China varies greatly. The primary care doctors in China have low levels of training, low job satisfaction, and high occupational stress; meanwhile, the application of information technology (IT) is fragmented [50]. This method is helpful to promote the application of openEHR and even EHR in developing countries, such as China.

Previous studies showed that using concept subnetwork structure could help us estimate semantic similarity and improve retrieval results $(\mathrm{P} @ 10=0.6)$ [28]. In this study, we selected $\mathrm{P} @ 5$, which was better than $\mathrm{P} @ 10$ as an evaluation metric as finally metrics and got $\mathrm{P} @ 5=0.883$ (Expansion Tems \& Top 3), on average. Our experiments also led to the following points worth noting:

\section{Calculating semantic relevance of queries to solve ambiguity}

In the medical domain, there is one vocabulary that is used by medical professionals more frequently than others, whereas patients often use alternative and lay terms or synonyms [51]. For example, 'medication item' and 'medicine item' referred to the same terms. Yang [29] proposed a graphical retrieval method to improve archetype retrieval performance and validate the method's feasibility. However, the method presented lacks the calculation of the semantic relevance of synonyms or homonyms for search terms. In this study, we introduced synonymlearning in NLP into openEHR innovatively to solve the retrieval errors caused by the lack of synonyms.

The essence of the NLP technology used in this article is to expand the search terms. Table 4 verifies this. Taking Low Level Set as an example, when the threshold is Top5, 360 expansion terms and 840 combination terms are expanded from 40 original search terms. Combination terms are a combination of the expansion terms. Their length is generally greater than that of expansion tems, and they also contain many grammatical error terms. Since the current search method provided by $\mathrm{HMC}$ is based on character matching, the longer the search term, the lower the search accuracy. From Table 6, we can see that the results of combiantion terms are lower than expansion terms on each set (Low level set: expansion terms MAP: 0.967, combination terms MAP: 0.567; Medium level set: expansion terms MAP: 0.883, combination terms MAP: 0.217; High level set: expansion terms MAP: 0.608, combination terms MAP: 0.167).

\section{Sorting retrieval results on semantic similarity}

Table 4 illustrates that for the same original search term, as the threshold (Top 3, 5 and 10) increases, the corresponding expansion and combination term will also increase. In the example of Medium Level Set, there were a total of 40 original search terms which generated 267 expansion terms at Top 3, 455 terms at Top 5 and 891 terms at Top 10. How to integrate the returned results and present them to the searcher is a problem. This study proposes a method to solve this problem by using NLP technology, specifically: calculating the semantic relevance between name of each result and the original search term and arranging them in descending order.

\section{The excessive increase in the number of search terms did not improve the search precision}

The core step of this method is the expansion of search terms, but the excessive increase does not lead to an increase on the search precision. According to Table 6, when the threshold of High Level Set is Top 3, AP has reached 0.637. In contrast, when the threshold is Top 10 , AP is only 0.575 . Although the difference between the two values is small, it is also worth noting. There are too many expansion terms and unnecessary errors are introduced. We need to choose an appropriate threshold, which in this study is Top 3.

This feasible study also has some limitations. In our experiment, we selected Wikipedia data as the expansion corpus, which led to a decline in the expansion performance of high medical professional level search terms. In the future, we can use different corpora for word expansion and compare their effects. Also, the abbreviations and acronyms lead to decreased readability and pose challenges for information retrieval [31]. In a future study, we will aim to resolve the problem of abbreviations in archetype retrieval tasks.

\section{Conclusion}

The purpose of this study is to promote the interoperability in the using of openEHR. To achieve that, we proposed an approach using NLP technology and dictionary (or corpus) to find synonyms as alternative terms for original search terms. We constructed three sets to test our approach. The P@5 was improved by 0.767 on average, compared to the baseline method (without using our approach). It is helpful to accelerate and advance healthrelated computing and EHR sharing.

\footnotetext{
Abbreviations

EHR: Electronic health record; NLP: Nature language processing; HMC: Healthcare modelling collaboration; CS-GAN: Category sentence generative adversarial network; MLSREN: Multi-level semantic representation enhancement network; DB: Ndeep belief network; UMLS: Unified medical language system; SNOMED-CT: Systematized nomenclature of medicine clinical terms; AP: Average precision; P@3: Precision at 3; P@5: Precision at 5; MAP: Mean average precision; Low Level Set: Test set with low medical professional level; Medium Level Set: Test set with medium medical professional level; High Level Set: Test set with high medical professional level.
} 


\section{Acknowledgements \\ Not applicable.}

\section{Authors' contributions}

WZ, BS conceived the study and developed algorithm. YY, JL and XD collected and preprocessed the data. FZ designe dexperimental and result analysis. BS and FZ carried out all the experimentand wrote the first draft of the manuscript. All authors read and approved the final manuscript.

\section{Funding}

This research is supported by the Chinese Academy of Medical Sciences (Grant\# 2018-12M-Al-006). The funder has no role in the study design, management, analysis and interpretation of data.

\section{Availability of data and materials}

The datasets used and analysed during the current study are available from the corresponding author on reasonable request.

\section{Declarations}

Ethics approval and consent to participate Not applicable.

\section{Consent for publication}

Not applicable.

\section{Competing interests}

The authors declare that they have no competing interests.

\section{Author details}

${ }^{1}$ Department of Information Center, Fuwai Hospital, National Center for Cardiovascular Diseases, Chinese Academy of Medical Sciences and Peking Union Medical College, No. 167 North Lishi Road, Xicheng District, Beijing 100037, China. ${ }^{2}$ Beijing Advanced Innovation Center for Biomedical Engineering, School of Biological Science and Medical Engineering, Beihang University, 37 Xueyuan Road, Haidian District, Beijing 100191, China. ${ }^{3}$ Fuwai Hospital, National Center for Cardiovascular Diseases, Chinese Academy of Medical Sciences and Peking Union Medical College, No. 167 North Lishi Road, Xicheng District, Beijing 100191, China. ${ }^{4}$ National Institute of Hospital Administration, National Health Commission, Building 3, yard 6, Shouti South Road, Haidian, Beijing 100044, China.

Received: 22 October 2020 Accepted: 8 June 2021

Published online: 26 June 2021

\section{References}

1. Murdoch TB, Detsky AS. The inevitable application of big data to health care. JAMA. 2013;309(13):1351-2.

2. Chaudhry B. Systematic review: impact of health information technology on quality, efficiency, and costs of medical care. Ann Intern Med. 2006;144(10):742-52.

3. Hillestad R, Bigelow J, Bower A, Girosi F, Meili R, Scoville R, Taylor R. Can electronic medical record systems transform health care? Potential health benefits, savings, and costs. Health Aff (Millwood). 2005;24:1103-17.

4. Patel KK, Nadel J. Improving the quality and lowering the cost of health care: medicare reforms from the national commission on physician payment reform. J Gen Intern Med. 2014;29(5):703-4.

5. Kennedy-Shaffer L. When the alpha is the omega: p-values, "substantial evidence", and the 005 standard at FDA. Food Drug Law J. 2017;72(4):595.

6. Verheij RA, Curcin V, Delaney BC, Mcgilchrist MM. Possible sources of bias in primary care electronic health record data use and reuse. J Med Intern Res. 2018;20(5):e185.

7. Fickenscher KM. President's column: interoperability - the $30 \%$ solution: from dialog and rhetoric to reality. J Am Med Inform Assoc JAMIA. 2013;3:593-4.

8. Saleem JJ, Flanagan ME, Wilck NR, Jim D, Doebbeling BN. The nextgeneration electronic health record: perspectives of key leaders from the US Department of Veterans Affairs. J Am Med Inform Assoc JAMIA. 2013;2013(e1):e175.

9. Roadmap D, Europe FOR. Semantic Interoperability for Better Health and Safer Healthcare. 2009.

10. Commision E. eHealth Action Plan 2012-2020-innvative healthcare for the 21st century. 2011.

11. Jianxing $H$, Sally $B$, Jie $X$, Jiming $X$, Zhou. The practical implementation of artificial intelligence technologies in medicine. Nat Med; 2019.

12. Alberto MC, da David MCWD, Santos MR, Alberto MJ, Montserrat R. Clinical information modeling processes for semantic interoperability of electronic health records: systematic review and inductive analysis. J Am Med Inform Assoc JAMIA. 2015;4:925-34.

13. Blobel B, Goosscn W, Brochhausen M. Clinical modeling-A critical analysis. Int J Med Informatics. 2014;83(1):57-69.

14. Edidin H, Bhardwaj V: HL7 Version 2. x Data Types. In: HL7 for BizTalk. Springer; 2014: 169-174.

15. Saripalle RK. Fast health interoperability resources (FHIR): current status in the healthcare system. Int J E-Health Med Commun. 2019;10(1):76-93.

16. Standards S. Health informatics-Electronic health record communication-Part 1: Reference model. 2006.

17. Standards B. Health Informatics-Electronic Healthcare Record Communication-Part 3: Distribution Rules.

18. Health informatics - Electronic health record communication-Part 2: Archetypes interchange specification (Endorsed by AENOR in October of 2007). 2007.

19. Beale T, Heard S, Kalra D, Lloyd D: OpenEHR architecture overview. The OpenEHR Foundation 2006, 7.

20. Costa CM, Menárguez-Tortosa M, Fernández-Breis JT. Clinical data interoperability based on archetype transformation. J Biomed Inform. 2011;44(5):869-80.

21. Wang L, Min L, Wang R, Lu X, Duan H. Archetype relational mapping-a practical openEHR persistence solution. BMC Med Inform Decis Mak. 2015;15(1):88

22. Role of OpenEHR as an open source solution for the regional modelling of patient data in obstetrics. J Biomed Inform. 2015.

23. Sundvall E, Nyström M, Karlsson D, Eneling M, Orman H. Applying representational state transfer (REST) architecture to archetype-based electronic health record systems. BMC Med Inform Decis Mak. 2013;13(1):57.

24. Min L, Tian Q, Lu X, Duan H. Modeling EHR with the openEHR approach: an exploratory study in China. BMC Med Inform Decis Mak. 2018;18(1):75.

25. Garde S, Knaup P, Hovenga EJS, Heard S. Towards semantic interoperability for electronic health records. Methods Inf Med. 2007;46(03):332-43.

26. Bernstein K, Tvede I, Petersen J, Bredegaard K. Can openEHR archetypes be used in a national context? The Danish archetype proof-of-concept project. Stud Health Technol Inform. 2009;150:147-51.

27. Garde S, Chen R, Leslie H, Beale T, McNICOLL I, Heard S. Archetype-based knowledge management for semantic interoperability of electronic health records. In: MIE: 2009; 2009. pp. 1007-11.

28. Yang L, Huang X, Li J. Discovering clinical information models online to promote interoperability of electronic health records: a feasibility study of OpenEHR. J Med Intern Res. 2019;21(5):576.

29. Min L, Tian Q, Lu X, An J, Duan H. An openEHR based approach to improve the semantic interoperability of clinical data registry. BMC Med Inform Decis Mak. 2018;18(Suppl 1):15.

30. Cohen MA. A survey of current work in biomedical text mining. Brief Bioinform. 2005;6(1):57-71.

31. Meystre SM, Savova GK, Kipper-Schuler KC, Hurdle JF. Extracting information from textual documents in the electronic health record: a review of recent research. Yearb Med Inform. 2008;17(01):128-44.

32. Chen G, Ye D, Xing Z, Chen J, Cambria E. Ensemble application of convolutional and recurrent neural networks for multi-label text categorization. In: 2017 International joint conference on neural networks (IJCNN): 2017; 2017

33. Guo J, Lu S, Han C, Zhang W, Wang J. Long text generation via adversarial training with leaked information. 2017.

34. Li Y, Pan Q, Wang S, Yang T, Cambria E. A generative model for category text generation. Inf Sci. 2018:S0020025518302366.

35. Younas M, Jawawi DNA, Ghani I. Extraction of non-functional requirement using semantic similarity distance. Neural Comput Appl. 2019;11:8989.

36. Jin $L$, Yang $Y$, He H. Multi-level semantic representation enhancement network for relationship extraction. Neurocomputing. 2020:403:282-93. 
37. Crimp R, Trotman A. ACM: refining query expansion terms using query context; 2018.

38. Chaturvedi I, Ong YS, Tsang IW, Welsch RE, Cambria E. Learning word dependencies in text by means of a deep recurrent belief network. Knowl Based Syst. 2016;108:144-54.

39. Huang Q, Yang Y, Cheng M. Deep learning the semantics of change sequences for query expansion. Softw Pract Exp. 2019;49(11):1600-17.

40. Yusuf N, Yunus MAM, Wahid N, Wahid N, Nawi NM, Samsudin NA. IEEE: enhancing query expansion method using word embedding. In: 2019 IEEE 9th international conference on system engineering and technology. 2019; pp. 232-235.

41. Pennington J, Socher R, Manning C. Glove: global vectors for word representation. In: Conference on empirical methods in natural language processing. 2014; 2014.

42. Liang Y, Zhang W, Yang K. Attention-based Chinese word embedding. In: International conference on cloud computing and security: 2018; 2018. pp. 277-87.

43. Liang C, Shao Y, Jing Z. Construction of a Chinese semantic dictionary by integrating two heterogeneous dictionaries: TongYiCi Cilin and HowNet. In: IEEE/WIC/ACM international joint conferences on web intelligence: $2013 ; 2013$.

44. Leroy $\mathrm{G}$, Chen H. Meeting medical terminology needs-the ontologyenhanced Medical Concept Mapper. IEEE Trans Inf Technol Biomed. 2001;5(4):261-70.
45. Koehn P, Knight K. Empirical methods for compound splitting. arXiv preprint CS/0302032; 2003.

46. Alfonseca E, Bilac S, Pharies S. Decompounding query keywords from compounding languages. In: Proceedings of ACL-08: HLT, short papers: 2008; 2008. pp. 253-56.

47. Zhao H, Cai D, Huang C, Kit C. Chinese word segmentation: another decade review (2007-2017). arXiv preprint arXiv:190106079; 2019.

48. Mikolov T, Sutskever I, Chen K, Corrado GS, Dean J. Distributed representations of words and phrases and their compositionality. In: Advances in neural information processing systems: 2013; 2013. pp. 3111-9.

49. Tay Y, Tuan LA, Hui SC. Multi-cast attention networks for retrieval-based question answering and response prediction; 2018.

50. Li X, Lu J, Hu S, Cheng KK, De Maeseneer J, Meng Q, Mossialos E, Xu DR, Yip W, Zhang H, et al. The primary health-care system in China. The Lancet. 2017;390(10112):2584-94.

51. Henriksson A, Moen H, Skeppstedt M, Daudaraviius V, Duneld M. Synonym extraction and abbreviation expansion with ensembles of semantic spaces. J Biomed Sem. 2014;5(1):6.

\section{Publisher's Note}

Springer Nature remains neutral with regard to jurisdictional claims in published maps and institutional affiliations.
Ready to submit your research? Choose BMC and benefit from:

- fast, convenient online submission

- thorough peer review by experienced researchers in your field

- rapid publication on acceptance

- support for research data, including large and complex data types

- gold Open Access which fosters wider collaboration and increased citations

- maximum visibility for your research: over 100M website views per year

At BMC, research is always in progress.

Learn more biomedcentral.com/submissions 